\title{
EL PRINCIPIO DE VOLUNTARIEDAD COMO PRESUPUESTO SUSTANTIVO DEL TRABAJO A TIEMPO PARCIAL
}

\author{
The Voluntary Principle Like Substantive Budget \\ of the Part-Time Work
}

\author{
Pilar Callau Dalmau \\ Doctora en Derecho \\ Profesora asociada del Departamento de Derecho de la Empresa \\ Universidad de Zaragoza
}

\section{ABSTRACT}

A pesar de que la figura del contrato a tiempo parcial exige además de una prestación de servicios regular realizada durante una jornada inferior a la ordinaria comparable, que la misma se haya acordado de una forma voluntaria, organismos internacionales advierten sobre el incremento y el excesivo peso que está cobrando el trabajo a tiempo parcial involuntario.

Esta naturaleza voluntaria recogida en diferentes ordenamientos supranacionales como una de las mayores preocupaciones de las leyes sobre el trabajo a tiempo parcial, por ser el factor que distingue este tipo de trabajo del subempleo visible, no es reconocida en el ordenamiento español como uno de los principios básicos en su regulación, hasta la modificación legislativa llevada a cabo por una transposición de las normas comunitarias, para la mejora del mercado de trabajo en relación con el trabajo a tiempo parcial y el fomento de su estabilidad. 
En este artículo, tras analizar la delimitación y los referentes normativos del principio de voluntariedad como elemento configurador del trabajo a tiempo parcial, se concreta su concurrencia en la extensión del contrato, desde su inicio y desarrollo hasta su finalización, y cómo su aplicación es imprescindible, para evitar la desnaturalización de la relación laboral adquirida.

Palabras clave: principio de voluntariedad, trabajo a tiempo parcial, relación laboral, subempleo, flexibilidad.

In spite of the fact that the figure of the part-time contract demands besides a regular provision of services realized during a day lower than the ordinary comparable one, that the same one has remembered a voluntary form, international organizations notice on the increase and the excessive weight that is receiving the part-time involuntary work.

This voluntary nature gathered in different supranational classifications like one of the major worries of the laws on the part-time work, for being the factor that distinguishes the part-time work of the visible underemployment, is not recognized in the Spanish classification as one of the basic beginning in his regulation, up to the legislative modification carried out by a transposition of the community procedure, for the improvement of the labor market in relation by the part-time work and the promotion of his stability.

In this article, after analyzing the delimiting and the normative modals of the beginning of wilfulness as element configurator of the part-time work, his concurrence makes concrete in the extension of the contract, from his beginning and development up to his ending, and how his application is indispensable, to avoid the denaturalization of the labor acquired relation.

Keywords: beginning of wilfulness, I work part-time, labor relation, underemployment, flexibility. 


\section{SUMARIO}

1. Introducción. 2. Referentes normativos. 2.1. Orden internacional. 2.2. Orden europeo. 2.3. Orden interno. 3. La concurrencia del principio de voluntariedad en el contrato a tiempo parcial. 3.1. Extensión a la formalización del contrato. 3.2. Extensión a la conversión de un trabajo a tiempo completo en un trabajo parcial y viceversa. 3.3. Extensión a la movilidad en el trabajo a tiempo parcial. 3.4. Extensión a las vertientes cualitativa y cuantitativa del tiempo de trabajo a tiempo parcial. 3.4.1. Horas complementarias. 3.4.1.1. Horas complementarias pactadas. 3.4.1.2. Horas complementarias no pactadas o de aceptación voluntaria. 4. Conclusiones. 5. Referencias bibliográficas.

\section{Introducción}

Asumiendo que la reducción de tiempo de trabajo es la referencia cuantitativa más característica y relevante del trabajo a tiempo parcial (Rafael, 1996: 58), que no hay que confundir con otras figuras afines en las que también se da una reducción de la jornada del trabajo ${ }^{1}$, motivada por razones personales de la persona trabajadora o manifestadas por la empresa (García-Perrote y Nieto, 2010: 25); la definición propuesta al principio de los años sesenta por la Organización Internacional del Trabajo (en adelante, OIT) del trabajo a tiempo parcial para

1 Dado que constituye una verdadera modalidad contractual, posee un régimen jurídico propio y no es una nueva variante reducida del contrato de trabajo a tiempo competo; tal como se ha pronunciado la jurisprudencia, justificando la consideración del trabajo a tiempo parcial como una verdadera modalidad contractual, al que no cabe identificar como un supuesto de reducción de jornada o de utilización reducida del tiempo de trabajo. Así se afirma en la STS de 14 de mayo de 2007, Sala Cuarta de lo Social (Rec. 85/2006), dónde la Sala señala que para calificar a una relación como contrato de trabajo a tiempo parcial no basta con que la reducción del tiempo de trabajo sea inferior a la jornada ordinaria a tiempo completo, sino que es preciso que la reducción de jornada sea voluntariamente adoptada con sujeción a la concreta modalidad de contrato a tiempo parcial, no integrando el contrato de que tratamos ni la reducción derivada del ejercicio de un derecho de la persona trabajadora, ni las limitaciones en trabajos con riesgo especial para la salud, dado que "no integran el contrato de que tratamos: la suspensión del contrato por causas económicas, técnicas, organizativas o de producción o derivadas de fuerza mayor [art. 47 ET]; la reducción derivada del ejercicio de un derecho del trabajador, por guarda legal o cuidado directo de familiar [art. 37.5 ET]; y las limitaciones en trabajos con riesgo especial para la salud [arts. 34.7, 36.1 y 37.1 ET; RD 1561/1995, de 21/Septiembre, sobre jornadas especiales de trabajo]" (FJ $3 .{ }^{\circ} 1$ ). 
diferenciarlo de otras formas de empleo como el trabajo ocasional o estacional y el trabajo temporal, es la del "trabajo efectuado de manera regular y voluntaria durante un período sensiblemente más corto de lo normal» (Jallade, 1985: 266).

Esta tipicidad del contrato a tiempo parcial, también se contempla a escala europea en el Acuerdo marco sobre el trabajo a tiempo parcial, celebrado en 1997 entre los interlocutores sociales de la Unión Europea (en adelante, UE); y aunque en el mismo no se define de manera expresa el trabajo a tiempo parcial, ya que deja su aplicación a las personas que trabajan a tiempo parcial con un contrato o una relación laboral tal y como se define en cada país de la UE, sí que prevé la posibilidad de excluir por razones objetivas a las que trabajen solamente de manera ocasional, si así lo acuerda el país de la UE en cuestión y sus interlocutores sociales.

Asimismo, este Acuerdo enuncia los principios generales y requisitos mínimos, tanto para garantizar la supresión de las discriminaciones contra las personas que trabajan a tiempo parcial y mejorar la calidad de este tipo de trabajo, como para facilitar su desarrollo sobre una base voluntaria y contribuir a la organización flexible del tiempo de trabajo, de una manera que tenga en cuenta las necesidades de las empresas y de sus plantillas.

De esta manera, la figura del contrato a tiempo parcial, exige el cumplimiento acumulativo de tres requisitos: además de una jornada inferior a la ordinaria comparable, es necesario que la prestación de servicios se haga de forma regular y que se haya acordado de forma voluntaria (García-Perrote y Nieto, 2010: 20).

Este concepto de voluntariedad, recogido en diferentes ordenamientos supranacionales como una de las mayores preocupaciones de las leyes sobre el trabajo a tiempo parcial, no es reconocido en el ordenamiento español como uno de los principios básicos en su regulación, hasta la modificación legislativa llevada a cabo mediante el Real Decreto-ley 15/1998, de 27 de noviembre, de Medidas Urgentes para la Mejora del Mercado de Trabajo en Relación con el Trabajo a Tiempo Parcial y el Fomento de su Estabilidad, con las que se pretende mejorar la calidad de este tipo de trabajo, garantizando tanto la supresión de toda discriminación de las personas que trabajan a tiempo parcial frente a las que lo hacen a tiempo completo, como el desarrollo de este tipo de trabajo sobre una base de naturaleza voluntaria.

Este aspecto, es el factor que distingue el trabajo a tiempo parcial del subempleo, tanto por escoger la persona trabajadora un régimen de trabajo a tiempo parcial deliberadamente, como por no tener que aceptar un horario reducido por no encontrar una colocación a tiempo completo. No hay que olvidar que el trabajo a tiempo parcial, históricamente en Espańa, ha aglutinado "todas las manifestaciones de lo que se considera precariedad", como la in- 
voluntariedad, la unilateralidad en la determinación de las condiciones de trabajo, la retribución minorada en su parte proporcional respecto a un trabajo a tiempo completo, la excesiva temporalidad de los contratos de trabajo, las duraciones de los mismos demasiado reducidas, el elevado índice de rotación, la concentración de este modelo de empleo en personas con una cualificación muy baja, en muchas ocasiones jóvenes, o la feminización de este clase de trabajo $y$, consecuentemente, las discriminaciones indirectas que se producen (Escudero,1997: 10-13).

En este sentido, se hace preciso subrayar que el subempleo denominado «visible», es el que guarda una relación directa con el trabajo a tiempo parcial involuntario, ya que se trabaja involuntariamente menos de la duración normal del trabajo y se está disponible para un trabajo adicional (De Villota, 1998: 138139) ${ }^{2}$; en contraposición con el subempleo «invisible» definido como «un concepto analítico ${ }^{3}$, que refleja una mala distribución de los recursos de mano de obra o un desequilibrio fundamental entre la mano de obra y los otros factores de producción (...) y cuyos sintomas característicos podrian ser el bajo nivel de los ingresos, el aprovechamiento insuficiente de las calificaciones y la baja productividad» (Bolle, 1997: 611-612).

Sobre esta cuestión, organismos internacionales han advertido en varias ocasiones, sobre el incremento y el excesivo peso que está cobrando el trabajo a tiempo parcial involuntario, que ya es un motivo de preocupación creciente en todos los países (OIT, 2014: 92-93). Respecto a España, un informe reciente de la OIT señala que, aunque en toda la UE, la involuntariedad del trabajo a tiempo parcial ha crecido algo, aquí, el trabajo parcial involuntario está pasando a ser un problema cada vez más apremiante a raíz de la crisis de 2008 (OIT, 2014: 92), ya que los datos de este aumento son mucho mayores que en el resto de la UE, tanto para hombres como para mujeres (CES, 2015: 251).

De hecho, el empleo involuntario a tiempo parcial como porcentaje del empleo a tiempo parcial total, supone un 61,9 por ciento — creciendo 28,6 puntos porcentuales desde el año 2007-, porcentaje que duplica con creces la media europea, donde el 27,7 por ciento de las personas no quieren este tipo de trabajo ${ }^{4}$.

En esta línea, los últimos datos del Instituto Nacional de Estadística (en adelante, INE) muestran que el trabajo a tiempo parcial, promovido por diferentes iniciativas en los últimos años y que, como es lógico, está peor remunerado, es

2 Contemplado en los mismos términos por el Instituto Nacional de Estadística (INE).

3 Cuyas normas estadísticas internacionales se adoptaron inicialmente en 1982, en la Resolución sobre estadísticas de la población económicamente activa, del empleo, del desempleo y del subempleo, en la decimotercera Conferencia Internacional de Estadísticos del Trabajo (CIET).

${ }^{4}$ Fuente: Eurostat (Última actualización: 17/01/2018). 
una modalidad forzada por la coyuntura socioeconómica española, ya que el trabajar a jornada parcial por no haber podido encontrar un trabajo de jornada completa, es aducido en Espańa por un 67,8 por ciento de hombres y un 59,8 por ciento de mujeres, mientras que en la UE-28, el porcentaje de mujeres que utilizan este tipo de contrato por este motivo es de un 24,6 por ciento 5 .

\section{Referentes normativos}

El alcance del principio de voluntariedad, antes de reconocerse en el ordenamiento jurídico español como uno de los principios básicos que configuran el marco jurídico del trabajo a tiempo parcial, se contempla tal como se ha indicado en el apartado anterior, en diferentes ordenamientos supranacionales como una de las mayores preocupaciones de las leyes sobre el trabajo a tiempo parcial, por ser el factor que distingue el trabajo a tiempo parcial del subempleo visible.

\subsection{Orden internacional}

En el plano internacional, el principio de voluntariedad se recoge expresamente en dos Convenios internacionales muy relevantes en lo que concierne al trabajo a tiempo parcial: por un lado, en el Convenio sobre la igualdad de oportunidades y de trato entre trabajadores y trabajadoras-trabajadores con responsabilidades familiares, 1981 (núm. 156) ${ }^{6}$; y por otro, en el Convenio sobre el trabajo a tiempo parcial, 1994 (núm. 175) ${ }^{7}$.

Mientras que en el primero se insta a adoptar todas las medidas compatibles con las condiciones y posibilidades nacionales, para permitir a las personas que trabajan con responsabilidades familiares el ejercicio de su derecho a elegir libremente su empleo, teniendo en cuenta sus necesidades en lo que concierne a las condiciones de empleo y a la seguridad social (art. 6); en el segundo, el principio de voluntariedad se recoge contemplando expresamente el trabajo a tiempo parcial.

5 Fuente: INEbase. Empleo (Fecha de consulta: 28/02/2018).

${ }^{6}$ Adoptado en Ginebra, en la 67.a reunión de la Conferencia General de la Organización Internacional del Trabajo, de 23 de junio de 1981, junto a la Recomendación sobre los trabajadores con responsabilidades familiares, 1981 (núm. 165), reemplazada por la Recomendación sobre el empleo de las mujeres con responsabilidades familiares, 1965 (núm. 123), adoptada en Ginebra, en la 49. ${ }^{a}$ reunión de la Conferencia General de la Organización Internacional del Trabajo, de 22 de junio de 1965.

7 Adoptado en Ginebra, en la 81.a reunión de la Conferencia General de la Organización Internacional del Trabajo, de 24 de junio de1994; junto a la Recomendación sobre el trabajo a tiempo parcial, 1994 (núm. 182), adoptada en Ginebra, en la 81. a reunión de la Conferencia General de la Organización Internacional del Trabajo, de 24 junio de1994. 
En este último instrumento jurídico, se promueve la adopción de medidas respecto a la promoción del trabajo a tiempo parcial, productivo y libremente elegido, siempre que se garantice su protección en toda la extensión del Convenio. Asimismo, se reivindica tanto una revisión de las disposiciones legislativas que dificulten la aplicación de esta modalidad contractual, como una especial atención en el marco de las políticas de empleo; haciendo hincapié en las necesidades de colectivos como las personas desempleadas, las que trabajan con responsabilidades familiares, las de edad avanzada, las discapacitadas y las que estén cursando estudios o prosigan su formación profesional (art. 9). También se contempla en el mismo, la voluntariedad en el caso de traslado de personas trabajadoras, de un trabajo a tiempo completo a un trabajo a tiempo parcial, o viceversa (art. 10).

\subsection{Orden europeo}

Dentro del ámbito europeo, hay que destacar como una contribución fundamental a la Estrategia Europea de Empleo ${ }^{8}$ en lo que al trabajo a tiempo parcial se refiere, el Acuerdo marco sobre el trabajo a tiempo parcial, celebrado el 6 de junio de 1997, por la Unión de Confederaciones de la Europa industrial y de empleadores (UNICE) — conocida actualmente como Business Europe-, el Centro Europeo de la Empresa Pública (CEEP) y la Confederación Europea de Sindicatos (CES), que figura en el anexo de la Directiva 97/81/CE del Consejo, de 15 de diciembre de $1997^{9}$.

La aplicación de las disposiciones que se contemplan en este Acuerdo, tiene como objetivos fundamentales, garantizar la supresión de las discriminaciones injustificadas contra las personas que trabajan a tiempo parcial, mejorar la calidad de este tipo de modalidad laboral, contribuir a la organización flexible del tiempo de trabajo de una manera que tenga en cuenta las necesidades de las empresas y de sus plantillas, y facilitar el desarrollo del trabajo a tiempo parcial sobre una base voluntaria (cláusula 1 ).

\footnotetext{
8 Cuyo objetivo principal es reducir significativamente el desempleo a escala europea. Para ello, se crea un marco de vigilancia y coordinación multilateral que incluye, un informe conjunto sobre el empleo anual, unas directrices de empleo que servirán de base para la elaboración de los Planes nacionales de acción por los Estados miembros, así como recomendaciones del Consejo de Ministros a los distintos Estados miembros, comprometiendo a estos sobre un conjunto de objetivos y metas comunes, articulados sobre cuatro pilares: la empleabilidad, el espíritu empresarial, la adaptabilidad y la igualdad de oportunidades. Todo esto, siguiendo el mandato del Tratado de Amsterdam, que en su art. $109 \mathrm{~N}$ contempla que "Los Estados miembros y la Comunidad se esforzarán, de conformidad con el presente título, por desarrollar una estrategia coordinada para el empleo, en particular para potenciar una mano de obra cualificada, formada y adaptable y mercados laborales con capacidad de respuesta al cambio económico (...)", y considerando las conclusiones del Consejo Europeo celebrado en Amsterdam en junio de 1997 y su Resolución sobre crecimiento y empleo de 16 de junio de 1997.

${ }^{9}$ DO L 014 de 20 de enero de 1998, pp. 9-14.
} 
Además, se refuerza el principio de voluntariedad como un elemento constituyente de la propia tipicidad del contrato a tiempo parcial, al disponer expresamente que «el rechazo de un trabajador a ser transferido de un trabajo a tiempo completo a un trabajo a tiempo parcial o viceversa, no debería por si mismo constituir un motivo válido de despido, sin perjuicio de la posibilidad de realizar despidos, de conformidad con las legislaciones, convenios colectivos y practicas nacionales, por otros motivos tales como los que pueden derivarse de las necesidades de funcionamiento del establecimiento considerado» (cláusula 5.2) ${ }^{10}$.

Con este Acuerdo alcanzado entre los interlocutores sociales europeos, no cabe duda que se clarifica y mejora los derechos del contrato a tiempo parcial, ya que además de garantizar la igualdad de trato y no discriminación de las personas que trabajan a tiempo parcial en relación con las que lo hacen a tiempo completo, y regular el acceso efectivo a la protección social de las personas contratadas a tiempo parcial, se ampara el principio de voluntariedad en el acceso al trabajo a tiempo parcial, que incorpora el derecho a no ser despedidas por negarse a ser transferidas desde un empleo a tiempo completo a otro a tiempo parcial, dejando a los Estados miembros la aplicación de disposiciones más favorables.

10 Esta cláusula por la que se fortalece la posición de las personas que trabajan a tiempo parcial, al imposibilitar la imposición unilateral por parte de la empresa, la conversión de un contrato a tiempo completo en un contrato a tiempo parcial o viceversa, ha sido interpretada recientemente por el Tribunal de Justicia de la UE (en adelante, TJUE), con la Sentencia del Tribunal de Justica de la Unión Europea (en adelante, STJUE), Asunto C-221/2013, de 15 de octubre 2014, que resuelve una petición de decisión prejudicial relativa a la interpretación del Acuerdo marco sobre el trabajo a tiempo parcial, en el marco de un litigio por la revocación unilateral de un régimen de trabajo a tiempo parcial, e imposición de uno a tiempo completo.

En la misma, se llega a una conclusión cuanto menos impactante, ya que el TJUE interpreta literalmente que «una situación en que el contrato laboral a tiempo parcial se transforma en contrato laboral a tiempo completo sin el acuerdo del trabajador afectado no es comparable con otra en que el contrato laboral a tiempo completo del trabajador se transforma en contrato laboral a tiempo parcial contra la voluntad de éste, pues la reducción del tiempo de trabajo no tiene las mismas consecuencias que su incremento, en particular, en el plano de la remuneración del trabajador, que constituye la contrapartida del trabajo" (Cuestión prejudicial 27), por lo que «la cláusula 5, apartado 2, debe interpretarse en el sentido de que no se opone, en circunstancias como las del litigio principal, a una normativa nacional en virtud de la cual el empleador puede disponer la transformación de un contrato laboral a tiempo parcial en contrato laboral a tiempo completo sin el acuerdo del trabajador afectado» (Cuestión prejudicial 28).

Lo que señala el Tribunal expresamente es que la situación de la trabajadora no es comparable con la contraria, en la que la reducción del tiempo de trabajo llevaría aparejada una consecuencia negativa para la trabajadora como es la reducción salarial proporcional a la de su tiempo de trabajo, de lo que se puede deducir, al menos esto es lo que mayoritariamente se interpreta por las voces más autorizadas, que esta es una sentencia que resuelve una cuestión puntual, y que podría haberse resuelto de manera contraria si el asunto hubiese sido la transformación de un contrato a jornada completa en un contrato a tiempo parcial, pero que en cualquier caso crea incertidumbre sobre el principio básico de voluntariedad en la contratación a tiempo parcial. 
Esto supone en definitiva que, fijados estos principios esenciales sobre los cuales se ha de basar la regulación del contrato a tiempo parcial, los mismos afectan directamente al desarrollo pertinente que sobre esta figura contractual se realice en el ordenamiento nacional.

\subsection{Orden interno}

En consecuencia, en nuestro ordenamiento jurídico, y tras la transposición de la Directiva 97/81/CE — que constituye una armonización con las leyes establecidas en el conjunto de países de la UE_, se plasman los principios básicos establecidos en el Acuerdo Marco sobre la figura del contrato de trabajo a tiempo parcial, en la redacción vigente del artículo 12 del Real Decreto Legislativo $2 / 2015$, de 23 de octubre, por el que se aprueba el texto refundido de la Ley del Estatuto de los Trabajadores (en adelante, ET).

En esta nueva configuración del marco jurídico del trabajo a tiempo parcial, los principios básicos que se contemplan son: la igualdad de trato y no discriminación de las personas que trabajan a tiempo parcial en relación con las que lo hacen a tiempo completo, sin perjuicio de la aplicación del principio de proporcionalidad cuando resulte adecuado; el principio de voluntariedad en el acceso al trabajo a tiempo parcial, que incorpora el derecho de la persona que trabaja a no ser despedido por negarse a ser transferido desde un empleo a tiempo completo a otro a tiempo parcial; y el acceso efectivo a la protección social de las personas contratadas a tiempo parcial; introduciendo para ello los elementos de corrección necesarios para adaptar el funcionamiento de las normas generales del sistema a las características específicas de este tipo de trabajo.

Esta modificación legislativa llevada a cabo mediante el Real Decreto-ley 15/1998, que se acomoda tanto a los compromisos establecidos a escala europea, como a los contenidos en el Acuerdo de Racionalización y Consolidación del Sistema de la Seguridad Social ${ }^{11}$ y en el Acuerdo Interconfederal para la Estabilidad en el Empleo ${ }^{12}$, también contempla que para identificar a un contrato como celebrado a tiempo parcial, deben concurrir tres aspectos concretos: la realización de una jornada inferior a la ordinaria comparable, la prestación de servicios de una forma regular y la voluntariedad en su ejecución ${ }^{13}$.

11 Firmado el 9 de octubre de 1996, por los sindicatos confederales CCOO y UGT y por el Gobierno.

12 De 28 de abril de 1997, en el que CEOE, CEPYME, CCOO y UGT apuestan por potenciar la contratación indefinida; favorecer la inserción laboral y la formación práctica de los jóvenes; especificar y delimitar los supuestos de utilización de la contratación temporal; y mejorar conjuntamente con el Gobierno el marco de la protección social del trabajo a tiempo parcial.

13 Extremo que se plasma en la STS de 14 de mayo de 2007 (Rec. 85/2006), al considerar la Sala como gran parte de la doctrina, que el mismo forma parte indiscutible de la compleja configuración legal del contrato a tiempo parcial (FJ 2.․2). 


\section{La concurrencia del principio de voluntariedad en el contrato a tiempo parcial}

Esta nota de voluntariedad que cobra gran importancia dentro de la relación laboral en todas las fases de la ejecución de un contrato a tiempo parcial, desde su inicio y desarrollo hasta su finalización (Valdés, 2002: 51-68), se desprende de diferentes artículos contemplados en el Código Civil (en adelante, CC) y en el ET, y entre los que cabe destacar los relacionados con la formalización del contrato, la conversión de un trabajo a tiempo completo en un trabajo parcial o viceversa, la movilidad, y la realización de horas complementarias.

\subsection{Extensión a la formalización del contrato}

En cuanto a la formalización del contrato a tiempo parcial, al igual que ocurre con todo contrato privado, rige el principio de voluntariedad, dado que de lo contrario, se iría en contra de lo previsto en el artículo 1256 del Código Civil que dispone que «la validez y cumplimiento de los contratos no puede dejarse al arbitrio de uno de los contratantes».

En este punto, hay que recalcar que siempre ha de hacerse por escrito, puesto que así se especifica tanto en su regulación ${ }^{14}$, como en la disposición legal que contempla la forma del contrato, y en la que este requisito, además de ser obligatorio para los contratos por tiempo determinado cuya duración sea superior a cuatro semanas, los de prácticas y aprendizaje, los contratos de trabajo a domicilio, los contratos para la realización de una obra o servicio determinado, y los de las personas contratadas en España al servicio de empresas españolas en el extranjero, también se extiende a los contratos celebrados a tiempo parcial ${ }^{15}$.

Esta exigencia formal que rompe con el principio espiritualista ${ }^{16}$, se establece legalmente como obligatoria en el caso de relaciones de trabajo que pue-

${ }_{14}$ Que dispone en la letra a) del apartado 4 del art. 12 ET que «el contrato, conforme a lo dispuesto en el artículo.8.2 de esta Ley, se deberá formalizar necesariamente por escrito», también regulado en la letra a) del art. 8 ET y el art.18 del Real Decreto 2317/1993, de 29 de diciembre, por el que se desarrolla los contratos en prácticas y de aprendizaje y los contratos a tiempo parcial.

15 Como se establecen en el apartado 2 del art. 8 ET; además el apartado 3 del art. 10 ET recoge otro supuesto, el del auxiliar o ayudante asociado a la persona que trabaja, en que se precisa la forma escrita.

${ }_{16}$ Que excluye que la forma pueda ser un requisito esencial, pero no un obstáculo para su relevancia a otros efectos, como se deriva del art. 1279 CC; es contraria al formalismo, conforme al que los contratos se perfeccionan por el mero consentimiento de las partes, sin necesidad de plasmar este consentimiento de una forma determinada, potenciando así la autonomía de la voluntad, plasmada en el principio de libertad de forma que rige nuestro ordenamiento jurídico, consagrado en el art.1278 CC, por el que "los contratos serán obligatorios, cualquiera que sea la forma en que se hayan celebrado, siempre que en ellos concurran las condiciones esenciales para su validez». De aplicación también en el Derecho del Trabajo, por el que las partes empresarial y trabajadora pueden elegir adquirir los compromisos que conlleva la relación laboral, verbalmente, por escrito o tácitamente, tal como se 
den ser negativas para los intereses de las personas trabajadoras, por poseer elementos particulares que las hace más vulnerables. Está prevista en casos como la formalización de los contratos formativos, los temporales, los contratos a domicilio, o los contratos a tiempo parcial, en aras de la tutela y protección de la parte trabajadora que rige en el Derecho del Trabajo.

Su naturaleza declarativa, que se traduce en un valor probatorio por contribuir a dar certidumbre al propio contrato y su contenido, no es constitutiva o ad solemnitatem ${ }^{17}$, por lo que su omisión no comporta la ineficacia del contrato, sino que pone en juego la presunción iuris tantum ${ }^{18}$ por la que se permite probar la inexistencia de los hechos, en el caso del contrato de trabajo a tiempo parcial, el del carácter parcial de los servicios, y si es caso, el de la temporalidad de los mismos, correspondiendo a la parte empresarial la carga de la prueba que destruya esta presunción.

La consecuencia jurídica por lo tanto de este incumplimiento formal, se traduce en la ineficacia del contrato de trabajo a tiempo parcial, pero no del contrato de trabajo en sí, ya que de no formalizarse por escrito, el contrato a tiempo parcial se presumirá celebrado a jornada completa y por tiempo indefinido ${ }^{19}$, salvo prueba en contrario que acredite su naturaleza temporal o el carácter a tiempo parcial de los servicios ${ }^{20}$.

Asimismo, el hecho de no formalizar por escrito el contrato de trabajo cuando este requisito sea exigible como es el caso del contrato a tiempo parcial,

desprende del art. 8.1 ET, dónde se establece que "el contrato de trabajo se podrá celebrar por escrito o de palabra. Se presumirá existente entre todo el que presta un servicio por cuenta y dentro del ámbito de organización y dirección de otro y el que lo recibe a cambio de una retribución a aquél. el acuerdo entre las partes podrá ser por escrito o de palabra».

17 Haciéndolo extensible a la regulación del tratamiento general de la forma en el derecho contractual común. Así lo avala la STS de 16 septiembre de 2014, Sala Primera (Rec. 969/2012), que fija como doctrina jurisprudencial que «el incumplimiento de la obligación de elevar a escritura pública el contrato de compraventa celebrado, conforme a lo dispuesto por el artículo 1280 del Código Civil, no es causa directa de resolución contractual» al amparo del art. 1124 del Código Civil.

18 Como da a entender la STSJ del País Vasco, de 11 de julio de 2000 (Rec.1548/2000), en la que en el caso de autos, los litigantes no tenían formalizado por escrito su contrato de trabajo a tiempo parcial en la fecha del despido, lo que hace entrar en juego la mencionada presunción legal favorable al carácter completo de la jornada estipulada, que únicamente cederá si se acredita cumplidamente que, en esa fecha, tenían pactada una jornada inferior.

19 A pesar de no especificarse en el desarrollo normativo de la letra a) del apartado 4 del art. 12 ET, la presunción de contrato celebrado por tiempo indefinido en caso de no formalizarse el contrato a tiempo parcial por escrito, sí se regula esta presunción expresamente en el desarrollo normativo relativo a la forma del contrato del ET, que en cualquier caso también se contempla en el apartado 1 del art. 9 del Real Decreto 2720/1998, de 18 de diciembre, por el que se presumirán celebrados por tiempo indefinido los contratos de duración determinada cuando no se hubiesen observado las exigencias de formalización escrita, salvo prueba en contrario que acredite su naturaleza temporal.

20 Último párrafo del apartado 2 del art. 8 ET. 
constituye una infracción grave ${ }^{21}$ que conlleva la oportuna sanción administrativa económica ${ }^{22 .}$

\subsection{Extensión a la conversión de un trabajo a tiempo completo en un trabajo parcial y viceversa}

Respecto a la posibilidad de convertir un trabajo a tiempo completo en un trabajo parcial y viceversa, se proclama de manera expresa el principio de voluntariedad en la conversión de este tipo de contrato, en el párrafo primero de la letra e) del apartado 4 del artículo 12 ET.

En esta disposición normativa, se establece que «la conversión de un trabajo a tiempo completo en un trabajo parcial y viceversa, tendrá siempre carácter voluntario para el trabajador, sin que pueda imponerse por lo tanto unilateralmente por el empresario, o como consecuencia de una modificación sustancial de condiciones de trabajo a que se refiere el articulo 41.1.a) ETi" ${ }^{23}$. De esta manera, la persona que trabaja no puede ser despedida ni sufrir ningún otro tipo de sanción o efecto perjudicial por el hecho de rechazar esta conversión, sin perjuicio de las medidas que, de conformidad con lo dispuesto en los artículos 51 y 52.c) ET se adopten.

Previsión legal de la que se desprende, primero, el carácter voluntario que tiene la transformación tanto de un trabajo a tiempo completo en un trabajo a tiempo parcial como de un trabajo a tiempo parcial a uno de tiempo completo; segundo, que una imposición unilateral por parte de la empresa nunca es posible, aunque sea ésta a causa de una modificación sustancial de condiciones de trabajo, por contravenir frontalmente la exigencia de voluntariedad en la novación del contrato a tiempo completo en un contrato a tiempo parcial ${ }^{24}$; y tercero, que de negarse la persona afectada, ésta no puede ser despedida o sancionada, sin perjuicio de las medidas que, de conformidad con lo dispuesto en los artículos 51 y 52.c) ET, puedan adoptarse por causas económicas, técnicas, organizativas o de producción.

Por tanto, no puede confundirse la modificación de la jornada a través del procedimiento del artículo $41 \mathrm{ET}$, sobre modificaciones sustanciales de las condiciones de trabajo cuando existan probadas razones económicas, técnicas, or-

${ }^{21}$ Regulada en el art. 7 del Real Decreto Legislativo 5/2000, de 4 de agosto, por el que se aprueba el texto refundido de la Ley sobre Infracciones y Sanciones en el Orden Social (TRLISOS).

22 Ibidem, letra b) del apartado 1 del art. 40.

${ }^{23}$ Que contempla las modificaciones sustanciales de condiciones de trabajo que afectan a la jornada de trabajo y sobre las que «la dirección de la empresa podrá acordar modificaciones sustanciales de las condiciones de trabajo cuando existan probadas razones económicas, técnicas, organizativas o de producción. Se consideraran tales las que estén relacionadas con la competitividad, productividad u organización técnica o del trabajo en la empresa».

${ }^{24}$ Como recuerda la STS de 14 de mayo de 2007 (Rec. 85/2006), avalada por la STS de 7 de octubre de 2011 (Rec. 144/2011). 
ganizativas o de producción, con la modificación del tipo de contrato a tiempo completo en uno a tiempo parcial, que es una novación contractual extintiva del viejo contrato y que sólo puede llevarse a cabo por un acuerdo entre las partes ${ }^{25}$, que en todo caso debe ser voluntaria ${ }^{26}$; con una reducción de la jornada laboral decidida unilateralmente por la empresa ante la disminución de la actividad empresariall $^{27}$.

Este hecho confirmado por la jurisprudencia, se extiende también a la interpretación de la normativa aplicable a la prestación por desempleo tras el cese en el trabajo, cuando éste se produce por una reducción de jornada derivada de la voluntad unilateral de la empresa. En este sentido, al no determinar esta imposición unilateral de la jornada reducida, la mutación del contrato de tiempo completo a uno a tiempo parcial, sino la mera reducción de la jornada del contrato a tiempo completo que persiste como tal dentro de esta categoría jurídica, no se está ante una novación unilateral de un contrato a tiempo completo en otro a tiempo parcial; teniendo por lo tanto la persona trabajadora que opta por la rescisión del contrato, derecho a la prestación por desempleo total, de la

25 Tal como sostiene la STC 213/2005, de 21 de julio de 2005, al afirmar que a raíz de la nueva regulación del contrato a tiempo parcial introducida en el art. 12 TRET por el Real Decreto-ley 15/1998, dejó de ser posible al amparo del art. 41 TRET una reducción de la jornada de trabajo en más de un tercio de su duración impuesta al personal en contra de su voluntad, dado que «una tal reducción de jornada implicaría la conversión del contrato a tiempo completo en un contrato "a tiempo parcial" y tal conversión 'tendrá siempre carácter voluntario para el trabajador, y no se podrá imponer de forma unilateral o como consecuencia de una modificación sustancial de condiciones de trabajo' [art. 12.4.e)] ET» (FJ 2. $\left.{ }^{\circ}\right)$.

26 Voluntariedad avalada por la STS de 14 de mayo de 2007 (Rec. 85/2006), dónde la Sala señala que para calificar a una relación como contrato de trabajo a tiempo parcial «no basta con que la reducción del tiempo de trabajo sea inferior a la jornada ordinaria a tiempo completo, sino que es preciso que la reducción de jornada sea voluntariamente adoptada con sujeción a la concreta modalidad de contrato a tiempo parcial (...)», a lo que ańade que "la imposición unilateral de jornada reducida (con carácter individual o colectivo) e incluso la modificación colectiva acordada de consuno con los representantes de los trabajadores, no determinan la mutación del contrato tiempo completoltiempo parcial, sino la mera reducción de la jornada en contrato a tiempo completo que persiste como tal categoría jurídica, pues la especifica modalidad de que tratamos (contrato a tiempo parcial) únicamente puede ser fruto de una conversión contractual que se instrumente por medio de una novación extintiva, que en todo caso es requirente de la voluntad concorde del trabajadon. De lo que se desprende que la transformación del contrato a tiempo completo en tiempo parcial necesita de la voluntad del personal afectado para que se produzca la necesaria novación extintiva del vínculo previo para acceder al nuevo contrato a tiempo parcial.

27 Ya que aplicando la anterior doctrina, la STS de 7 de octubre de 2011 (Rec. 144/2011), que sí exige el principio de voluntariedad para la conversión del art. 12.4.e) del ET, reitera que «de no darse esa conversión, al aplicarse en el caso una reducción de la jornada de la jornada, decidida unilateralmente por la empresa ante la disminución de la actividad empresarial, no supone una vulneración de lo previsto en el articulo 12.4 e) ET, desde el momento que esa decisión empresarial y la ausencia de conformidad de las trabajadoras no determinaban la transformación del contrato a tiempo completo en otro a tiempo parcial, tal y como se afirma en la sentencia recurrida, máxime cuando la media adoptada tenia el carácter de temporal, aunque ciertamente no se identificase el tiempo durante el que habría de producir efectos". 
misma manera que acaece con las extinciones voluntarias contempladas en el artículo $50 \mathrm{ET}^{28}$.

En esta línea, cabe destacar que, al igual que no cabe una reducción de la jornada laboral decidida unilateralmente por la empresa ante la disminución de la actividad empresarial, tampoco cabe una ampliación de la misma, ni cuando exista un presupuesto convencional genérico de que la jornada pueda ampliarse temporalmente cuando se den los supuestos de la contratación temporal establecidos en el convenio, ni siquiera, en el caso de una modificación colectiva al respecto acordada con los representantes del personal, por exigir este hecho el acuerdo entre las dos partes del contrato, respetando de esta manera la autonomía de la voluntad individual de la parte trabajadora ${ }^{29}$; por lo que si esta se niega a modificar la jornada, no puede ser despedida o sancionada, sin perjuicio de las medidas que, de conformidad con lo dispuesto en los artículos 51 y 52.c) ET, puedan adoptarse por causas económicas, técnicas, organizativas o de producción.

28 Así se contempla en la STSJ de Aragón, de 20 de abril de 2015 (Rec. 221/2015), que tiene su apoyo en la doctrina legal que emana de la STS de 14 de mayo de 2007 (Rec. 85/2006), reiterada después en lo sustancial en otros pronunciamientos similares, como la STS de 7 de octubre de 2011 (Rec. 144/2011), y en la que estimando el recurso de suplicación y revocando la sentencia recurrida, condena al Servicio Público de Empleo Estatal a abonar al actor la prestación por desempleo solicitada tras la extinción por su parte del contrato de trabajo al no haber aceptado la reducción de su jornada, ya que "nadie puede ser obligado a trabajar de forma distinta a la pactada en el contrato (...) y, al igual que ocurre con las extinciones contractuales voluntarias ampradas en el art. 50 ET, no enerva el derecho a dicha prestación»(FD 4. $\left.{ }^{\circ}\right)$.

29 Como proclama a este respecto, la STS 12 de mayo de 2015 (Rec. 153/2014), resolviendo la demanda interpuesta por las Federaciones Estatales de Comercio, Hostelería y Turismo de CCOO y UGT solicitando la nulidad de varios artículos del Convenio Colectivo sectorial de Grandes Almacenes, de ámbito estatal, siendo parcialmente estimada por la Sentencia de 13 de noviembre de 2013, dictada por la Sala de lo Social de la Audiencia Nacional, recaída en el procedimiento n. ${ }^{\circ}$ 278/2013 $y$, posteriormente, recurrida en casación por un lado, por los sindicatos accionantes, y por otro, por la Federación de Asociaciones Sindicales (FASGA) y la Asociación Nacional de Grandes Empresas de Distribución (ANGED).

$\mathrm{Su}$ pronunciamiento se realiza respecto a uno de los preceptos del convenio colectivo que son objeto de controversia, en el que se establece que en el contrato de trabajo a tiempo parcial se estará a lo dispuesto en el art. $12 \mathrm{ET}$, precisando en su apartado A3 que «La jornada inicialmente contratada podrá ampliarse temporalmente cuando se den los supuestos que justifican la contratación temporal. En la ampliación deberán concretarse las causas de la ampliación temporal»; ante el que la Sala entiende que "dicho precepto convencional constituye una invasión de la esfera de la autonomía de la voluntad» (FD 2., primer párrafo), y que además «se parte, pues, de una sumisión expresa y una remisión de igual clase al art 12 ET, al que se reconoce, por ello, como norma de referencia por su carácter de derecho necesario, cuyo apartado 4 regula el contrato a tiempo parcial, que no contempla, en ninguno de sus subapartados, la ampliación temporal de jornada en los términos a que se refiere el precepto convencional sino que en su apartado e) en la redacción vigente en el momento de interposición de la demanda y de dictarse sentencia (la actual entró posteriormente en vigor el 22 de diciembre de 2013 y fue introducida por el artículo 1.1 del Real Decreto-ley 16/2013, de 20 de diciembre) disponía que 'la conversión de un trabajo a tiempo completo en un trabajo a tiempo parcial y viceversa tendrá siempre carácter voluntario para el personal y no se podrá imponer de forma unilateral' (...)» (FD 2.․, segundo párrafo). 
Esta regulación se adecúa en parte, a la dispuesta en la cláusula 5.2 del Acuerdo marco sobre el trabajo a tiempo parcial, que figura en el anexo de la Directiva 97/81/CE, apuntada con anterioridad, "pudiendo los Estados miembros y/o los interlocutores sociales mantener o introducir disposiciones más favorables que las previstas en el presente Acuerdo» (cláusula 6).

\subsection{Extensión a la movilidad en el trabajo a tiempo parcial}

En lo referente a la movilidad voluntaria en el trabajo a tiempo parcial, el párrafo segundo de la letra e) del apartado 4 del artículo $12 \mathrm{ET}$, además de imponer un deber de información a la empresa sobre la existencia de puestos de trabajo vacantes para facilitar la movilidad voluntaria en el trabajo a tiempo parcial, insta a los convenios colectivos al establecimiento de los procedimientos oportunos para que la empresa informe a su plantilla sobre la existencia de puestos de trabajo vacantes; para que las personas interesadas y siempre de manera voluntaria soliciten, o la conversión de un trabajo a tiempo completo en un trabajo a tiempo parcial y viceversa; o para que en los casos de las personas que trabajan a tiempo parcial, soliciten un incremento del tiempo de trabajo.

Es importante precisar que, en cualquier caso, estas solicitudes que deben ser tomadas en consideración en la medida de lo posible por la empresa, si se deniegan, han de ser notificadas obligatoriamente por escrito y resueltas siempre de manera motivada.

\subsection{Extensión a las vertientes cualitativa y cuantitativa del tiempo de trabajo a tiempo parcial}

En cuanto al establecimiento de la jornada parcial, dado que en nuestra sociedad el trabajo a tiempo completo es el considerado como habitual frente al trabajo a tiempo parcial; es la jornada completa la que se configura como jornada de trabajo típica, y por tanto, sirve de marco referencial para establecer la jornada parcial, no sólo en la regulación legal, sino también en la colectiva del trabajo (Beltrán, 2000: 140-150).

La determinación de esta vertiente cuantitativa o número total de horas durante las que se prestan los servicios en el trabajo a tiempo parcial, y la de la vertiente cualitativa o distribución de las mismas a lo largo de un periodo determinado de tiempo (Basterra, 2014: 2), además de ser uno de los rasgos distintivos que definen al contrato a tiempo parcial por conferirle un régimen jurídico distinto; es una condición laboral que trasciende del ámbito individual de la relación de trabajo, al incidir también en otros aspectos laborales como la organización productiva de la empresa, la política de empleo, y, desde luego los aspectos salariales directamente relacionados con la jornada, siempre protagonista en la negociación colectiva (González, Sequeira y Tejedor, 2001: 320). 
De la concreción de estas dos vertientes se desprenden diferentes tipos de trabajo a tiempo parcial. Además del trabajo a tiempo parcial estándar, conocido como trabajo a tiempo parcial «horizontal», en el que la prestación laboral de la persona que trabaja a tiempo parcial se corresponde con una actividad diaria que temporalmente se desarrolla de manera semejante a la de una que lo hace a tiempo completo, salvo que esa prestación es reducida en el tiempo trabajado, sin que se vea afectada por ninguna suspensión; cabe también el denominado trabajo a tiempo parcial "vertical», que aunque se ejecuta solamente en ciertos días de la semana, del mes o del año, se identifica con el horizontal, debido a que en ambos supuestos la relación laboral está determinada por la continuidad en el alta de la persona que trabaja durante todo el período del contrato (Barcelón, 2013: 35-36); así como el «mixto» en el que la reducción afecta a horas al día y al año ${ }^{30}$.

En consecuencia, en el trabajo a tiempo parcial, el establecimiento del horario es un elemento constitutivo del contrato, que junto a la jornada, representa el tiempo de prestación de servicios (Vicedo, 2008: 228.).

Su concreción de manera voluntaria es fundamental, debido a que las peculiaridades de los elementos que conforman el trabajo a tiempo parcial, lo hacen muy susceptible de un alto grado de flexibilidad que, como reflejo de la nueva organización del trabajo y las necesidades de empleo, debe estar en el centro de las negociaciones entre la dirección de las empresas y los representantes del personal, para adaptarla al modelo europeo de sociedad (Comisión de las Comunidades Europeas, 1993:18), y evitar que desemboque en una disponibilidad completa del personal contratado bajo esta modalidad por parte de la empresa ${ }^{31}$.

\subsubsection{Horas complementarias}

En relación con el principio de voluntariedad y la vertiente cuantitativa apuntada anteriormente, una cuestión esencial en materia de flexibilidad, en cuanto a la ordenación del tiempo de trabajo a tiempo parcial se refiere, es el de las horas complementarias ${ }^{32}$.

Su concepto es algo más que una mera definición, dado que, junto con los elementos identificadores propios y delimitadores de esta figura, contiene elementos normativos propios que resultan centrales en su regulación (RodríguezPiñero y Calvo, 2000: 39 y ss.). Como su propio nombre indica, son horas

30 Calificativos a los que también hace alusión la STS, Sala de lo Social, de 14 de mayo de 2007 (Rec. 85/2006), relacionados con la distribución del tiempo de trabajo en una relación de trabajo a tiempo parcial, diferenciando la distribución horizontal (reducción de horas al día), vertical (número de días a la semana, al mes o al ańo) o mixta (horas al día y al ańo).

31 En este sentido, la STSJ de Valencia, de 3 de diciembre de 1996 (Rec. 923/1996).

32 Sobre las que el RDL 16/2013, introduce algunos cambios importantes en lo que a su regulación se refiere. 
adicionales a las horas ordinarias pactadas en el contrato a tiempo parcial que complementan la jornada hasta un límite máximo, el de la jornada completa, conforme a una serie de reglas contempladas en el apartado 5 del artículo 12 ET, y donde además de las horas complementarias pactadas, cobra especial importancia una nueva variante de jornada adicional, la opción de las horas complementarias voluntarias (Martínez, 2014: 101).

De este modo, dentro de las horas complementarias se establece una distinción entre las pactadas, de realización obligatoria para el personal cuando se haya firmado el preceptivo pacto de horas complementarias ${ }^{33}$; y las voluntarias, que únicamente pueden ser ofrecidas por la empresa en los contratos que tengan una duración indefinida. La diferencia entre las horas complementarias pactadas y las horas complementarias voluntarias radica en "la mecánica en virtud de la cual deviene obligatoria su realización: en ambos casos, lógicamente, la realización de las horas ha de aceptarse por el trabajador, pero mientras en las pactadas, la aceptación es general y previa, de modo que el pacto da cobertura a la obligación de realizar las que en el futuro se exijan por el empresario, en las de aceptación voluntaria, la aceptación es especifica y posterior al ofrecimiento empresarial, de modo que dicha aceptación se proyecta sobre horas concretas y perfectamente determinadas en cantidad y ubicación temporal» (Gómez, 2014: 118).

En este sentido, también es necesario precisar que la diferencia con las horas extraordinarias, reside en que estas son horas ańadidas que realizan las personas que trabajan a jornada completa ya que la misma no es susceptible de complementarse; pues hay que recordar que, tras la entrada en vigor del Real Decretoley 16/2013, de 20 de diciembre, de medidas para favorecer la contratación estable y mejorar la empleabilidad de los trabajadores, en los contratos a tiempo parcial, se prohíbe ${ }^{34}$ la posibilidad de realizar horas extraordinarias ${ }^{35}$, salvo en

\footnotetext{
33 Y que la disposición transitoria única del Real Decreto-ley 16/2013, prevé que el régimen de horas complementarias pactado con anterioridad a la entrada en vigor de este real decreto-ley continúe siendo de aplicación en los contratos vigentes a dicha fecha salvo que las partes acuerden modificarlo en los términos establecidos en la nueva regulación, y que los periodos de prueba que se hubieran concertado con anterioridad a la entrada en vigor de este real decreto-ley continúen rigiéndose por la normativa legal o convencional conforme a la que se celebraron.

$34 \mathrm{Al}$ respecto, la STS de 11 de junio de 2014 (Rec. 1039/2013), dictamina que "cuando un trabajador contratado a tiempo parcial realiza efectivamente una jornada superior a la pactada, y ese exceso no pueda ser calificado como tiempo u hora 'complementaria', bien sea porque tal cuestión esté fuera de discusión, bien sea porque no se hayan cumplido los requisitos previstos al efecto (...), todo lo que supere en esa materia el contenido del pacto, constituyen horas extraordinarias y como tal han de ser retribuidas, al margen o con independencia de la prohibición legal para efectuarlas".

35 Prohibición introducida por la letra c) del apartado uno del art. 1 del Real Decreto-ley 16/2013, por la que se modifica la letra c) del apartado 4 del art.12 ET. Estas horas no se permitieron en estos contratos hasta la reforma laboral de febrero de 2012, prohibidas previamente con la reforma laboral de 1998, en línea con la regulación del art. 12 de la R182-Recomendación sobre el trabajo a tiempo parcial (núm. 182) sobre el trabajo a tiempo parcial, que pese a admitir el exceso de jornada sobre el horario acordado, indica en su párrafo 2 que «en la medida de lo posible, las modifica-
} 
los casos en los que su realización sea imprescindible para prevenir o reparar siniestros y otros daños extraordinarios y urgentes ${ }^{36}$, en cuyo caso, su cotización es la establecida legalmente para la cotización adicional para este tipo de horas extraordinarias ${ }^{37}$.

En cuanto a la regulación del régimen de las horas complementarias ${ }^{38}$, prevista en los párrafos $2 .^{\circ}$ y $3 .^{\circ}$ de la letra c) del apartado 4 , y apartado 5 del artículo $12 \mathrm{ET}$, contempla que las mismas, suponen una adición o prolongación a las horas ordinarias pactadas en el contrato a tiempo parcial (art. 12.5 ET), equiparándose con éstas de tal manera que, el tratamiento de las horas complementarias efectivamente realizadas es como el de las ordinarias tanto en materia retributiva como de Seguridad Social, teniéndose en cuenta por lo tanto a efectos de períodos de carencia y de bases reguladoras de las prestaciones ${ }^{39}$ (art. 12.5.i ET).

\subsubsection{Horas complementarias pactadas}

En lo que a las horas complementarias pactadas se refiere, hay que puntualizar que para su realización, es necesario formalizar expresamente por escrito un pacto de horas complementarias de naturaleza constitutiva, pues de lo contrario se supone inexistente; y que si este pacto firmado cumple todos los requisitos legalmente establecidos, pero no consta en modelo oficial $^{40}$, no afecta a su exis-

ciones de los horarios convenidos y el trabajo en exceso de dichos horarios deberia ser objeto de restricciones $y$ de un preaviso".

36 Apartado 3 del art.35 ET.

37 En el art. 106 de la Ley 3/2017, de 27 de junio, de Presupuestos Generales del Estado para el año 2017, se establece que los tipos generales de cotización en el Régimen General para la cotización adicional por horas extraordinarias cuando sean motivadas por fuerza mayor, el 14 por ciento, del que el 12 por ciento será a cargo de la empresa y el 2 por ciento a cargo de la persona que trabaja.

38 Figura constituida con la entrada en vigor del Real Decreto-Ley 15/1998, donde se regula la posibilidad de pactar horas complementarias sobre el número de las ordinarias fijadas, hasta un 15 ó 30 por 100 de éstas, según los casos, con la misma remuneración.

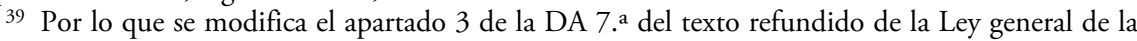
Seguridad Social, aprobado por Real Decreto Legislativo 1/1994, de 20 de junio (LGSS) —actual art. 246 del Real Decreto Legislativo 8/2015, de 30 de octubre-, sobre cotización en estos contratos; y el art. 9 del RD 1620/2011, sobre la relación laboral especial del servicio del hogar familiar. En este caso, la cotización se equipara a la de una hora ordinaria, por lo que su tipo de cotización es, según el art. 106 de la LPGE, en donde se establecen los tipos generales de cotización en el Régimen General, para las contingencias comunes el 28,30 por ciento, siendo el 23,60 por ciento a cargo de la empresa y el 4,70 por ciento a cargo de la persona que trabaja.

40 Contemplado en el Real Decreto-Ley 15/1998, por el que se autoriza al Director general del Instituto Nacional de Empleo para aprobar los modelos de contrato a tiempo parcial y de pacto de horas complementarias a que se refieren respectivamente la letra a) del apartado 4 y la letra a) del apartado 5 del artículo 12 ET, en la redacción dada por este Real Decreto-ley, en su DF 1.a.2. En este sentido, en el art. 5 del Anexo I sobre la relación de datos obligatorios a comunicar a los servicios públicos de empleo de la ORDEN TAS/770/2003, de 14 de marzo, se regula la comunicación del contenido de los contratos de trabajo y de sus copias básicas a los Servicios Públicos de Empleo, así como los datos obligatorios de la comunicación de horas complementarias. 
tencia, considerándose por lo tanto válido a todos los efectos (Rodríguez-Piñero y Calvo, 2000: 77).

Este pacto de naturaleza voluntaria, dado que debe ser consensuado por las partes, una vez hecho efectivo, es de obligatorio cumplimiento si así se lo requiere la empresa, ya que "podrá acordarse en el momento de la celebración del contrato a tiempo parcial o con posterioridad al mismo, pero constituirá, en todo caso, un pacto especifico respecto al contrato» (art. 12.5. a ET); teniendo en cuenta que aunque su realización puede ser requerida por la empresa, su ejecución aunque posible, no siempre es efectiva (art.12.5.c ET).

Ahora bien, el personal contratado a tiempo parcial, mediante un preaviso de 15 días, una vez cumplido un año desde la celebración del pacto de horas complementarias, puede renunciar al mismo quedando sin efecto, de darse las siguientes circunstancias: la atención de las responsabilidades familiares por razones de guarda legal, por necesidades formativas, siempre que se acredite la incompatibilidad horaria, y por incompatibilidad con otro contrato a tiempo parcial (art. 12.5.e ET).

En cuanto al número de horas que se debe hacer constar en el pacto de horas complementarias, al considerarse este aspecto contenido mínimo, la omisión de su concreción, acarrea sin más la nulidad del pacto por quedar indeterminado el objeto del mismo (art. $1273 \mathrm{CC}$ ). En este caso, la negativa a su realización no es constituyente de ninguna conducta laboral sancionable.

En este aspecto, si una persona contratada a tiempo parcial realiza efectivamente una jornada superior a la pactada, todo lo que supere en esa materia el contenido del pacto, constituye horas extraordinarias y como tal han de ser retribuidas, al margen o con independencia de la prohibición legal para efectuar$\operatorname{las}^{41}$. Y aunque en algunos casos cabe solicitar el reconocimiento y consolidación de la jornada real efectuada por la persona trabajadora, por ser superior a la contractualmente prevista, para que se dé un reconocimiento de la misma, es preciso que el exceso de jornada pretendida pueda considerarse como regular, y se deduzca en cómputo anual ${ }^{42}$.

41 Tal como se contempla en la STS, Sala 4. ${ }^{\text {a }}$, de lo Social, de 11 de Junio de 2014 (Rec. 1039/2013).

42 Como avala la STSJ Cataluña Sala de lo Social, de 16 de abril de 2014 (Rec. 96/2014), por la que "el hecho de haber realizado solo durante seis meses una jornada a tiempo completo por la vía de sumar a la jornada parcial pactada una serie de horas complementarias, impide apreciar la consolidación de una condición más beneficiosa en los términos expuestos, teniendo en cuenta que, como se razona en la sentencia, la jornada completa que realizaron lo fue en cómputo semanal, no anual" (FD 2. ${ }^{\circ}$. Además "Tampoco el hecho de que puntualmente la suma de la jornada ordinaria más las horas complementarias haya alcanzado la jornada ordinaria a tiempo completo lleva aparejada como consecuencia la conversión del contrato en otro a tiempo completo, pues lo que dice el artículo 12.5 del Estatuto de los Trabajadores al regular las horas complementarias es que el trabajador puede negarse a realizar aquellas que excedan de las pactadas, sin que por ello pueda ser sancionado» (FD 3.०.). 
Estas horas complementarias pactadas que pueden realizarse, no pueden exceder del 30 por ciento ${ }^{43}$ de las horas ordinarias de trabajo objeto del contrato; aunque los convenios colectivos pueden establecer otro porcentaje máximo, que, en ningún caso, debe ser inferior al citado 30 por ciento ni exceder del 60 por ciento de las horas ordinarias contratadas (párrafo 2..$^{\circ}$ del art. 12.5.c ET); manteniéndose la posibilidad de que la negociación colectiva altere esta cifra, pero sólo para incrementarla, prohibiéndose su rebaja por la vía convencional.

En este sentido, una precisión importante es que, para evitar prácticas fraudulentas, que conviertan un contrato a tiempo parcial en uno a tiempo completo, se establece que la suma total de las horas ordinarias y complementarias, incluidas las pactadas previamente y las voluntarias, no puede exceder del límite legal que define este contrato (párrafo 3. ${ }^{\circ}$ del art. 12.4.c ET), es decir, el de la jornada de trabajo de una persona que trabaja a tiempo completo comparable, debiéndose respetar los límites legales en materia de jornada y descansos obligatorios (art. 12.5.h ET).

Estas horas complementarias pueden realizarse tanto por las personas que trabajan temporalmente ${ }^{44}$ como por las que lo hacen de forma indefinida, con un único requisito, que la jornada semanal en cómputo anual alcance un mínimo de diez horas (art.12.5.b ET).Y en lo que al plazo de preaviso para realizarlas se refiere, el personal debe conocer el día y la hora de realización de las horas complementarias pactadas con un preaviso mínimo de tres días, salvo que en el convenio colectivo se establezca un plazo de preaviso inferior ${ }^{45}$ (art.12.5.d ET), precisando que sólo pueden rebajar el plazo, no ampliarlo.

Si se da algún incumplimiento en estas reglas, ya sea en el pacto de horas complementarias, o en las condiciones de realización de las mismas, la negativa a la realización de las mismas, aunque estas hayan sido pactadas, no constituye conducta laboral sancionable ${ }^{46}$ (art. 12.5.f ET); y de sancionarse laboralmente por su negativa, la imposición de un eventual despido, «debería recibir la calificación judicial de nulidad en base a la vulneración de derechos fundamentales que tiene lugar en todos estos casos (ya sea desde el punto de vista de su libertad personal — artículo 10.1 CE—, ya a la tutela judicial efectiva —art. 24.1 CE—) cuando el despido se produce como acto de represalia empresarial al trabajador por el ejercicio

43 Límite que antes de la reforma era del 15 por ciento.

${ }^{44}$ La posibilidad de realizar horas complementarias sólo era posible antes de la reforma, para las personas con contratos indefinidos.

45 Plazo que antes de la reforma, salvo que se estableciese otra cosa en el convenio, era de siete días.

46 Teniendo además en cuenta que la transgresión de las normas y los límites legales o pactados en materia de horas complementarias da lugar a la infracción grave prevista en el apartado 5 del art. 7 TRLISOS. 
legitimo de sus derechos", si es que previamente el hecho ha sido denunciado ante la Inspección de Trabajo y Seguridad Social (Sirvent, 2016: 16).

En este sentido, si no hay irregularidad alguna ni en el pacto de horas complementarias ni en las condiciones de realización de las mismas, la negativa a realizar las horas complementarias comprometidas en el pacto de considerarse grave, puede constituir causa de sanción disciplinaria, e incluso de despido disciplinario por "faltas injustificadas de asistencia al trabajo", o por "transgresión de la buena fe contractual» (Sirvent, 2016: 16).

\subsubsection{Horas complementarias no pactadas o de aceptación voluntaria}

Junto a las horas complementarias de origen pactado de antemano, y por lo tanto de ejecución obligatoria cuando sean solicitadas por la empresa si no se da ninguna irregularidad, para los contratos a tiempo parcial, se da carta de naturaleza a otras horas complementarias, las no pactadas o "voluntarias», cuya realización cabe independientemente de la formalización o no de un pacto de horas complementarias, al no haber prohibición expresa alguna en su regulación ${ }^{47}$. En este caso, de producirse una negativa a la realización de estas horas no constituye conducta laboral sancionable (art. 12.5.g ET).

Su régimen jurídico es de aplicación en los contratos a tiempo parcial de duración indefinida con una jornada de trabajo de, al menos, 10 horas semanales en cómputo anual, que la empresa puede ofrecer al personal, en cualquier momento. Su realización, que en ningún caso puede superar el 15 por ciento de las horas ordinarias contratadas, y que puede ampliarse por convenio colectivo hasta un 30 por ciento, no se computa a efectos de los límites establecidos para las horas complementarias pactadas.

A este respecto, es muy importante precisar que, en el caso de contratos a tiempo parcial de carácter temporal o con jornada inferior a la exigida legalmente, de no existir pacto de horas complementarias, o sobrepasar el número de horas pactadas, la realización de horas complementarias voluntarias, las convierte en horas extraordinarias ilegales —en el caso de ser accidentales - o, en un motivo de novación contractual —en el caso de ser habituales - al modificarse la jornada de la persona trabajadora tras incorporar estas horas complementarias voluntarias, "pudiendo ello dar lugar a que el contrato se considere celebrado incluso a tiempo completo. E idénticos efectos cabe atribuir a la realización de horas complementarias voluntarias por encima del tope máximo legal o convencional permitido» (Sirvent, 2016: 22).

47 Aunque hay autores que señalan todo lo contrario, porque "no existiendo dicho pacto, el trabajador no se ha mostrado disponible a realizar ninguna hora fuera de la jornada ordinaria", como Lousada (2014: 11). 
Dicho esto, se deduce que, además de crearse con el pacto de horas complementarias, lo que coloquialmente se denomina una bolsa horaria, que permite a la empresa asegurarse un número de horas, del 30 por ciento de la jornada pactada u ordinaria, ampliable al 60 por ciento por convenio colectivo, y que puede o no solicitar que sean realizadas por el personal en función de las necesidades de la empresa; si también el personal tiene contratos indefinidos a tiempo parcial con jornadas no inferiores a 10 horas semanales, la empresa puede ofrecer horas complementarias de aceptación voluntaria, cuyo número no puede ser superior al 15 por ciento ampliable hasta el 30 por ciento por convenio colectivo, respecto de la jornada ordinaria pactada, teniendo presente que estas horas son independientes del límite establecido para las horas complementarias pactadas.

El resultado es que, si una persona contratada a tiempo parcial con una jornada de veinte horas semanales, firma un pacto de horas complementarias y a la vez acepta otro de horas complementarias de aceptación voluntaria, ambos con el límite mínimo establecido legalmente, puede llegar a trabajar veintinueve horas semanales, si la empresa le requiere esas nueve horas de la bolsa horaria. Ahora bien, en caso de que por convenio se estableciesen los límites máximos tanto para las horas complementarias como para las complementarias de aceptación voluntaria, esta bolsa horaria pasa a ser de dieciocho horas semanales, por lo que la jornada puede llegar a treinta y ocho horas semanales a disposición de la empresa.

\section{Conclusiones}

La figura del trabajo a tiempo parcial ha sido objeto de regulación tanto por la OIT, como por las instituciones de la UE, con el fin, entre otros aspectos conexos, de concretar su extensión, promover su protección social y garantizar tanto la supresión de toda discriminación de las personas que trabajan a tiempo parcial frente a las que lo hacen a tiempo completo, como el desarrollo de este tipo de trabajo sobre una base de naturaleza voluntaria.

En nuestro ordenamiento jurídico, las últimas actuaciones reformistas llevadas a cabo sobre la configuración del trabajo a tiempo parcial y los principios que ordenan su marco jurídico, lejos de legislarse por iniciativa propia, se han ido acomodando a la regulación internacional y europea, e imponiendo tras sucesivas declaraciones de nulidad de las reglas de aplicación; para garantizar esta forma de trabajo vinculada a la flexibilidad, preservar los derechos sociales de las personas que trabajan dentro de su ámbito de aplicación, y evitar en último término, que la parcialidad involuntaria se afiance como un elemento estructural en el mercado de trabajo. 
La aplicación de estas normas de referencia por su carácter de derecho necesario, salvaguarda entre otros, el principio de voluntariedad que se hace imperativo en todas las fases del contrato a tiempo parcial, debiéndose adoptar para ello, las medidas necesarias para una promoción del trabajo a tiempo parcial, productivo y libremente elegido, ante la debilidad contractual de los colectivos en los que la utilización de esta figura es más habitual.

En este sentido, al constituir este principio un derecho individual irrenunciable y en consecuencia, un presupuesto sustantivo de la ordenación del trabajo a tiempo parcial, la ausencia o no concurrencia de este elemento en el mismo, desnaturaliza la relación contractual adquirida.

\section{Referencias bibliográficas}

Barcelón Cobedo, Susana (2013), Trabajo a tiempo parcial y Seguridad Social (Con las reformas introducidas por el RDL 11/2013), Tirant lo Blanch, Valencia.

Basterra Hernández, Miguel (2014), «La distribución de la jornada de trabajo en España», en Revista Internacional y Comparada de Relaciones Laborales y Derecho del empleo, Volumen 2, N.o 1.

Beltrán Felipe, Reyes (2000), «Las mujeres y el trabajo a tiempo parcial en España. Elementos para su análisis», en Cuaderno de Relaciones Laborales, N.o 17.

Bolle, Patrick (1997), «El trabajo a tiempo parcial, ¿libertad o trampa?», en Revista Internacional del Trabajo, Vol. 116, N.o 4.

Comisión de las Comunidades Europeas (1993), Crecimiento, competitividad y empleo. Retos y pistas para entrar en el siglo XXI — Libro Blanco-, Oficina de Publicaciones Oficiales de las Comunidades Europeas, Luxemburgo.

Consejo Económico y Social (2015), Economía, Trabajo y Sociedad. Memoria sobre la situación socioeconómica y laboral. España 2014, Consejo Económico y Social. España, Madrid.

De Villota, Paloma (1998), Las mujeres y la ciudadania en el umbral del siglo XXI, Ediciones Complutense, Madrid.

Escudero Rodríguez, Ricardo (1997), «Adaptabilidad y causalidad de la contratación temporal en la negociación colectiva posterior a la reforma", en Relaciones Laborales, N.o 2 .

García-Perrote Escartín, Ignacio y Nieto Rojas, Patricia (2010), «Aspectos laborales del contrato a tiempo parcial», en Revista jurídica de Castilla y León, n. ${ }^{\circ} 22$.

Goméz Abelleira, Fco. Javier (2014), «La reforma del contrato de trabajo a tiempo parcial», en AA.VV. Las reformas laborales y de Seguridad Social. De la Ley 11/2013, de 26 de julio, al Real Decreto-Ley 3/2014, de 28 de febrero, Lex Nova, Valladolid.

González Ortega, Santiago, Sequeira de Fuentes, Marcial y Tejedor Redondo, Luis (2001), Derecho del Trabajo de la Empresa, Colex, Madrid.

Jallade, Jean-Pierre (1985), Europa a tiempo parcial, Ministerio de Trabajo y Seguridad Social, Madrid.

Lousada Arochena, José Fernando (2014), «El trabajo a tiempo parcial en el Real Decreto-Ley 16/2013, de 20 de diciembre», en Nueva Revista Española de Derecho del Trabajo, n. ${ }^{\circ} 162$. 
Martínez Moreno, Carolina (2014), «¿Puede ser el contrato a tiempo parcial un verdadero instrumento de política de empleo", en Revista Derecho Social y Empresa, N.o 2.

Organización Internacional del Trabajo (2014), España: Crecimiento con empleo, Oficina Internacional del Trabajo, Ginebra.

Rafael Porras, Alfredo (1996), Contrato de trabajo a tiempo parcial, Ediciones Jurídicas Cuyo, Mendoza.

Rodríguez-Piñero Royo, Miguel C., y Calvo Gallego, Fco. Javier (2000), Las horas complementarias y su pacto en el contrato a tiempo parcial, Tirant Lo Blanch, Valencia.

Sirvent Hernández, Nancy (2016), «El régimen jurídico de la jornada complementaria en el contrato de trabajo a tiempo parcial tras las modificaciones introducidas por el Real Decreto-Ley 16/2013, de 20 de diciembre», en Trabajo y derecho: nueva revista de actualidad y relaciones laborales, $\mathrm{n} .^{\circ} 15$.

VALDÉs DAL-RÉ, Fernando (2002), «El trabajo a tiempo parcial: la (im)posible convivencia entre flexibilidad y seguridad», (1) y (H), en Relaciones Laborales: Revista Crítica de Teoria y Práctica, Vol. H.

Vicedo Cañada, Luisa (2008), El trabajo a tiempo parcial: Régimen sustantivo, La Ley, Madrid.

\section{Fuentes primarias}

\section{Orden supranacional}

Directiva 97/81/CE del Consejo, relativa al Acuerdo marco sobre el trabajo a tiempo parcial, de 15 de diciembre de 1997.

Convenio sobre el trabajo a tiempo parcial, 1994 (núm. 175).

Recomendación sobre el trabajo a tiempo parcial, 1994 (núm. 182).

Convenio sobre la igualdad de oportunidades y de trato entre trabajadores y trabajadorastrabajadores con responsabilidades familiares, 1981 (núm. 156).

Recomendación sobre los trabajadores con responsabilidades familiares, 1981 (núm. 165).

Recomendación sobre el empleo de las mujeres con responsabilidades familiares, 1965 (núm. 123).

\section{Orden interno}

Ley 3/2017, de 27 de junio, de Presupuestos Generales del Estado para el año 2017.

Real Decreto Legislativo 8/2015, de 30 de octubre, por el que se aprueba el texto refundido de la Ley General de la Seguridad Social.

Real Decreto Legislativo 2/2015, de 23 de octubre, por el que se aprueba el texto refundido de la Ley del Estatuto de los Trabajadores.

Real Decreto-ley 16/2013, de 20 de diciembre, de medidas para favorecer la contratación estable y mejorar la empleabilidad de los trabajadores.

Real Decreto 1620/2011, sobre la relación laboral especial del servicio del hogar familiar.

Orden TAS/770/2003, de 14 de marzo, por la que se desarrolla el Real Decreto 1424/2002, de 27 de diciembre, por el que se regula la comunicación del contenido de los contratos de trabajo y de sus copias básicas a los Servicios Públicos de Empleo.

Real Decreto 1424/2002, de 27 de diciembre, por el que se regula la comunicación del contenido de los contratos de trabajo y de sus copias básicas a los Servicios Públicos de Empleo, y el uso de medios telemáticos en relación con aquélla. 
Real Decreto Legislativo 5/2000, de 4 de agosto, por el que se aprueba el texto refundido de la Ley sobre Infracciones y Sanciones en el Orden Social.

Real Decreto 2720/1998, de 18 de diciembre, por el que se desarrolla el artículo 15 del Estatuto de los Trabajadores en materia de contratos de duración determinada.

Real Decreto-ley 15/1998, de 27 de noviembre, de medidas urgentes para la mejora del mercado de trabajo en relación con el trabajo a tiempo parcial y el fomento de su estabilidad.

Real Decreto 1561/1995, de 21 de septiembre, sobre jornadas especiales de trabajo.

Real Decreto 2317/1993, de 29 de diciembre, por el que se desarrolla los contratos en prácticas y de aprendizaje y los contratos a tiempo parcial.

Real Decreto de 24 de julio de 1889 por el que se publica el Código. Civil.

\section{Sentencias}

STJUE de 15 de octubre 2014 (Asunto C-221/2013).

STC 213/2005, de 21 de julio de 2005.

STS de 12 de mayo de 2015 (Rec. 153/2014).

STS de 11 de Junio de 2014 (Rec.1039/2013).

STS de 16 septiembre de 2014 (Rec. 969/2012).

STS de 11 de junio de 2014 (Rec. 1039/2013).

STS de 7 de octubre de 2011 (Rec. 144/2011).

STS de 14 de mayo de 2007 (Rec. 85/2006).

STSJ de Aragón, de 20 de abril de 2015 (Rec. 221/2015).

STSJ de Cataluña, de 16 de abril de 2014 (Rec. 96/2014).

STSJ del País Vasco, de 11 de julio de 2000 (Rec.1548/2000).

STSJ de Valencia, de 3 de diciembre de 1996 (Rec. 923/1996).

SAN de 13 de noviembre de 2013 (N.o 278/2013. 